\title{
Increase in Adiposity of Type 2 Diabetes Patients following Withdrawal from Therapeutic Exercise
}

\author{
Adeniyi, A.F., ${ }^{1}$ Sanya, A.O., ${ }^{1}$ Fasanmade, A.A., ${ }^{2}$ Uloko, A.E. ${ }^{3}$ \\ ${ }^{1}$ Department of Physiotherapy, Faculty of Clinical Sciences, College of Medicine, University of Ibadan, Ibadan, Nigeria \\ ${ }^{2}$ Department of Medicine, University College Hospital, Ibadan, Nigeria \\ ${ }^{3}$ Department of Medicine, Aminu Kano Teaching Hospital, Kano, Nigeria \\ Correspondence \\ A.F. Adeniyi, Department of Physiotherapy, Faculty of Clinical Sciences, College of Medicine, University of Ibadan, \\ Ibadan, Nigeria・Email: adeniyifatai@yahoo.co.uk
}

\begin{abstract}
SUMMARY
In order to achieve optimum health status, Type 2 Diabetes (T2D) patients are usually encouraged to undergo regular and consistent therapeutic exercises. This study investigated whether the gains of exercises on adiposity variables of T2D patients are maintained within a few weeks of withdrawal from exercise and whether it was significantly related to the age of the patient and the duration of diagnosis of T2D.

Forty-threeT2D patients aged between 30 and 64 years were recruited for this study. They were placed on a combination of endurance and strengthening exercises for 12 weeks. The participants were thereafter observed for another 12 weeks without exercises. The adiposity variables measured in both phases of the study include: body mass index (BMI), waist circumference (WC), waist-hip-ratio (WHR) and percent-body-fat (PBF).

Significant improvements $(\mathrm{P}<0.05)$ for all the variables were achieved after 12 weeks of therapeutic exercises. The variables, however, showed significant relapses $(\mathrm{P}<0.05)$ within six weeks of withdrawal of exercise and these were significantly related $(\mathrm{P}<0.05)$ to both the age of the patient and the duration of diagnosis.

The endurance and strengthening exercises improved the adiposity variables of theT2D patients, but the gains began to decline within six weeks of withdrawal from the exercises. The increase in adiposity was more as the age and duration of diagnosis of the patients increased.
\end{abstract}

KEY WORDS: weight maintenance, body composition, diabetes mellitus

\section{INTRODUCTION}

Formerly, diabetes was seen mostly in developed nations, but the disease is becoming commonplace in developing countries, where lifestyle practices have begun to emulate those of developed societies (King and Herman, 1998). With the modernization taking place, the lifestyle in many sub-Saharan African countries, particularly in urban areas, is similar to those in many Western countries, with the related epidemiological changes (Kengne et al, 2005). Generally, a number of factors are implicated in the pathogenesis of diabetes. Obesity contributes significantly to the burden of diabetes and other chronic health conditions (Kengne et al, 2005). Adiposity and level of physical activity are also significant and independent determinants of insulin sensitivity and secretion. A high degree of adiposity and a small proportion of time spent in physical activity are associated with low insulin sensitivity and high insulin secretion (Snitker et al, 2007). Low insulin sensitivity and high insulin secretion have been identified as precursors of T2D (Snitker et al, 2007).

Lifestyle intervention is associated with dramatic reductions in visceral fat as well as smaller but still significant decreases in subcutaneous fat, body weight, body mass index (BMI) and waist circumference (WC) in both men and women (Fujimoto et al, 2007). Diet and endurance exercises have been proven to improve BMI, body composition and body fat distribution, thus delaying or preventing T2D in individuals with impaired glucose tolerance (Liao et al, 2002). Modest weight loss, dieting or dieting and exercise resulted in similar improvements in total abdominal fat, subcutaneous adipose tissue and glycaemic status in postmenopausal women with T2D (Giannopoulou et al, 2005). These reports demonstrate the importance of exercise in the treatment of people with T2D.

Though therapeutic exercises play a complimentary 
role in the treatment of $\mathrm{T} 2 \mathrm{D}$ and its related disorders, there is sparse literature on how long the benefits attained on adiposity in T2D patients are maintained when therapeutic exercise is withdrawn. Studies designed to reduce body adiposity in individuals with $\mathrm{T} 2 \mathrm{D}$ are of great importance as body weight and body fat distribution are risk factors for diabetes. In non-diabetic individuals, weight-loss experts have argued that losing excess weight during treatment is the first of many steps needed to sustain weight loss, but maintaining the lost weight is the hard part of obesity treatment for most dieters (Craig, 2007). The age of the patient and duration of the diabetes have been shown to have an effect on the course of the disease and its complications (Donaghue et al, 2003; Scanlon et al, 2005). It is, however, uncertain how the age of the patient and duration of the diagnosis are related to adiposity in T2D patients, especially during the period of withdrawal from exercises. Based on these considerations, the aim of this modified crossover study was to investigate: 1)the effect of withdrawal from therapeutic exercise on adiposity, and 2) the relationship between the age of the patient, the duration of diagnosis and adiposity variables of T2D patients after withdrawal from exercise.

\section{METHOD}

Forty-three patients with T2D attending the Specialty (Diabetic) Clinic of the Aminu Kano Teaching Hospital (AKTH), Kano, Nigeria, met the eligibility criteria and volunteered to participate in this study. To qualify for inclusion in the study the participant must: (a) be diagnosed with diabetes by a physician, (b) consent to measurements, (c) be willing to give informed consent, and (d) must participate in the follow-up phase of the study. The study was conducted at the outpatient Physiotherapy Clinic of AKTH, Kano.

The Ethical Committee on Research of AKTH, Kano, approved the study. The consent of the physicians attending to these patients was also obtained. The study comprised two research phases spanning a period of 24 weeks, namely: the Exercise Phase (EP) of 12 weeks duration, and the Follow-Up Phase (FUP), also spanning 12 weeks.

In the EP, the participants received outlined therapeutic exercises in addition to the drug and/or diet regimen that might have been prescribed for them earlier. This included 3 sessions of endurance and strengthening exercises per week, with at least a day of rest in between for twelve consecutive weeks; making 36 sessions.

There was no exercise intervention for the FUP. Participants were, however, encouraged to comply with their prescribed drugs and diet. The FUP included two visits by the participants within the twelve weeks for reassessment - at the end of the $6^{\text {th }}$ and $12^{\text {th }}$ weeks, which coincided with the $18^{\text {th }}$ and $24^{\text {th }}$ weeks of the entire study.

\section{Adiposity Measurements}

The following data on the adiposity variables of the participants were recorded at baseline and subsequently at the end of the $6^{\text {th }}, 12^{\text {th }}, 18^{\text {th }}$ and $24^{\text {th }}$ weeks in both the EP and FUP.

1. Weight, height and body mass index (BMI): Body weight was measured in kilograms with a weighing scale, height was measuredwith a wall-mounted height meter in meters and BMI was calculated as weight in kilograms dividedby height in meters squared (Snitker et al, 2007).

2. Waist circumference (WC), hip circumference (HC) and waist-hip ratio (WHR): The waist was defined as the midpoint between the highest point of the iliac crest and the lowest point of the costal margin at the mid-axillary line. Waist circumference was measured with an inelastic tape in centimetres. Hip circumference was measured at the level of the greater femoraltrochanters in centimetres. The waist-hip ratio is the waist circumference divided by the hip circumference (Fujimoto et al, 2007).

3. Percent body fat (PBF): An Omron BF306 body fat monitor (Martin et al, 2001) was used to measure the percentage body fat.

The WHR and WC were used as measures of abdominal (central) adiposity, while BMI and PBF were used as measures of general adiposity (Wannamethee et al, 2005).

\section{Therapeutic Exercises}

The duration of each exercise session was 50 - 60 minutes. This included warm-up and cool-down exercises of 5 minutes each. The participants pedalled a bicycle ergometer, starting with an intensity of $60 \%$ of heart rate reserve (HRR); i.e. [0.6X (Heart Rate max - Heart Rate res $)+$ Heart Rate rest $_{\text {I }}$ for 20 minutes. This was broken into 2 parts of 10 minutes each, with a 5 -minute break. In addition, they did 2 rounds of brisk walking with full arm swings, up and down the length of the gymnasium (10m long), till they 
were exhausted. A minimum of 3 minutes rest was observed between the 2 laps. Exercises to strengthen the flexors and extensors of both the upper and lower limbs were also carried out. Sixty percent of the individual's one repetition maximum (1RM) was determined and used to strengthen each group of muscles (Sigal et al, 2004). Participants performed 2 sets of 10 repetitions, with a recovery time of 2 minutes between each set.

\section{Progression of Exercises}

The initial intensity of $60 \%$ of HRR in the bicycle ergometry was increased to $65 \%$ and $70 \%$ at the beginning of the $5^{\text {th }}$ and $9^{\text {th }}$ weeks of the study, respectively. New $1 \mathrm{RM}$ was determined every fortnight and $60 \%$ of that was utilized thereafter for the strengthening exercises.

\section{Data Analysis}

Mean and standard deviations were computed to describe the participants' ages, duration of diagnosis and the variables studied. General linear model repeated measures were generated to compare the variables in the various assessment periods. Scheffe's post-hoc analyses were computed to locate areas of significance. Pearson's product moment correlation coefficients were calculated to reveal the relationship between the components of adiposity, age of participants and duration of diagnosis. Significance level was set at $95 \%$.

\section{RESULTS}

The participants were between $30-64$ years, with the mean age and duration of diagnosis being $47.93 \pm 9.86$ and 5.29 \pm 4.39 years respectively. General linear model repeated measures show significant differences $(\mathrm{P}<0.05)$ for the components of adiposity that were measured at the different assessment periods (table 1). Scheffe's post-hoc analyses revealed significant improvements $(\mathrm{P}<0.05)$ between the baseline and the $12^{\text {th }}$ week adiposity variables. The means of adiposity variables in the follow-up phase (from the $12^{\text {th }}$ through the $18^{\text {th }}$ to the $24^{\text {th }}$ week) revealed increases during the withdrawal period. From the post-hoc analyses, these increases were significant for all the adiposity variables between the $12^{\text {th }}-18^{\text {th }}$ and $18^{\text {th }}-24^{\text {th }}$ weeks; except for $\mathrm{HC}$, which did not witness a significant relapse until much later, between the $18^{\text {th }}$ and $24^{\text {th }}$ weeks. During the follow-up phase, when the relapse occurred, bivariate correlations (table 2) show direct significant relationships $(\mathrm{P}<0.05)$ between the age of the participants and each of $\mathrm{BMI}{ }^{\circledR}=0.72$ ) and $\left.\mathrm{WC}{ }^{\circledR}=0.47\right)$; and duration of diagnosis and each of WHR ${ }^{\circledR}=0.52$ ) and WC ${ }^{\circledR}=0.59$ ). Within the same period, however, significant inverse relationships $(\mathrm{P}<0.05)$ were observed between the age of the participants and each of WHR $\left.{ }^{\circledR}=-0.61\right)$ and $\mathrm{PBF}{ }^{\circledR}=$ -0.43) and between duration of diagnosis and PBF ${ }^{\circledR}=-$ $0.55)$.

Table 1. Comparison of adiposity variables of participants during the exercise and follow-up phases revealing increase in the variables during follow-up

\begin{tabular}{|c|c|c|c|c|c|c|}
\hline Adiposity & Baseline & $12^{\text {th }}$ wk & $18^{\text {th }}$ wk & $24^{\text {th }}$ wk & $\mathbf{F}$ & P-value \\
\hline Wt $(\mathrm{kg})$ & $83.93 \pm 14.60$ & $66.76 \pm 8.92$ & $71.74 \pm 11.56$ & $82.18 \pm 8.31$ & 40.243 & $<0.05$ \\
\hline BMI $\left(\mathrm{kg} / \mathrm{m}^{2}\right)$ & $34.84 \pm 7.76$ & $27.74 \pm 5.37$ & $29.85 \pm 8.21$ & $34.18 \pm 6.32$ & 63.584 & $<0.05$ \\
\hline $\mathrm{WC}(\mathrm{cm})$ & $103.90 \pm 13.09$ & $91.04 \pm 9.73$ & $96.23 \pm 9.88$ & $101.90 \pm 11.56$ & 211.561 & $<0.05$ \\
\hline $\mathrm{HC}(\mathrm{cm})$ & $107.41 \pm 11.58$ & $106.34 \pm 13.11$ & $106.60 \pm 8.34$ & $106.94 \pm 8.90$ & 302.172 & $<0.05$ \\
\hline WHR & $0.96 \pm 0.04$ & $0.85 \pm 0.07$ & $0.89 \pm 0.02$ & $0.95 \pm 0.06$ & 27.645 & $<0.05$ \\
\hline PBF $(\%)$ & $39.4 \pm 7.39$ & $25.03 \pm 5.74$ & $27.15 \pm 6.44$ & $28.66 \pm 6.64$ & 17.782 & $<0.05$ \\
\hline
\end{tabular}

*All mean changes from one period to another were significant $(\mathrm{P}<0.05)$ except $\mathrm{HC}$ where it was significant only between $18^{\text {th }}$ and $24^{\text {th }}$ week. 
Adiposity in Type 2 Diabetes Patients following Withdrawal from Therapeutic Exercise

Table 2. Correlations between adiposity variables and each of age of participant and duration of diagnosis during the follow-up period

\begin{tabular}{|c|c|c|c|c|c|c|c|c|}
\hline & Age & Ddiag & WT & BMI & WC & $\mathrm{HC}$ & WHR & $\mathrm{PBF}$ \\
\hline Age & 1.00 & $* 0.65$ & $* 0.68$ & $* 0.72$ & $* 0.47$ & 0.21 & ${ }^{*}-0.61$ & $*-0.43$ \\
\hline DDiag & & 1.00 & $* 0.71$ & -0.16 & ${ }^{*} 0.59$ & -0.32 & $* 0.52$ & $*-0.55$ \\
\hline WT & & & 1.00 & $* 0.88$ & $* 0.56$ & $* 0.47$ & $* 0.68$ & $* 0.71$ \\
\hline BMI & & & & 1.00 & $* 0.68$ & 0.37 & $* 0.48$ & $* 0.53$ \\
\hline WC & & & & & 1.00 & $* 0.71$ & $* 0.63$ & $* 0.48$ \\
\hline $\mathrm{HC}$ & & & & & & 1.00 & $*-0.41$ & 0.26 \\
\hline WHR & & & & & & & 1.00 & ${ }^{*} 0.58$ \\
\hline PBF & & & & & & & & 1.00 \\
\hline
\end{tabular}

Notes: *Significant $(\mathrm{P}<0.05)$

WT - weight; BMI - body mass index; WC - waist circumference; HC - hip circumference; WHR - waist-hip-ratio; PBF - percent body fat; DDiag. duration of diagnosis.

\section{DISCUSSION}

The principal findings of this study were that the benefits of therapeutic exercises on adiposity in T2D patients were not sustained during the period of withdrawal from exercises. It was also discovered that the age of the participants and duration of diagnosis were related to the increase observed in the adiposity variables, with the exception of hip circumference. Although the achieved benefits of exercises on adiposity relapsed during the withdrawal phase, we noticed that the mean of the variables after 12 weeks of withdrawal did not reach the baseline level. This signifies that when exercises are withdrawn from T2D patients, after 12 weeks of moderate, 50-60 minutes, thrice weekly therapeutic exercises, the achieved benefits are still felt albeit minimally for at least 12 weeks. How much of these benefits are retained, however, would depend on how old the patient is or how long the patient has suffered T2D.

The fact that the participants in this study gained weight, thereby increasing their BMI and other body composition variables during the follow-up period, may be because they withdrew from the exercise sessions. This supports the finding that high levels of physical activity are necessary for successful weight maintenance (Klem et al, 1997). Evidence also suggests that the level of physical activity to be undertaken to maintain weight must be substantial to prevent weight regain (Schoeller et al, 1997). In a group of overweight and obese women, the addition of 275 minutes per week of physical activity, in addition to a reduction in energy intake, was important in sustaining a weight loss of more than $10 \%$ (Jakicic et al, 2008).
Reporting on weight gain over a period of time in nondiabetic individuals, Devlin et al (2000) claimed that a comprehensive behavioural weight-control programme, comprising improved eating habits, lifestyle change and increased exercise, is widely viewed as the treatment of choice for overweight and moderately obese individuals. The authors however claimed that the treatment effects were not maintained over time.

Therapeutic exercise has many beneficial effects on adiposity, and withdrawal from it could bring about a relapse in the benefits. Lowered blood pressure and blood lipids and improvement in glycaemic control are some of the benefits that can accrue from maintenance of weight loss, and these often keep individuals motivated to continue with their weight-loss efforts (Craig, 2007). In a group of Japanese-Americans with impaired glucose tolerance, it was found that small changes in overall adiposity as measured by BMI could be associated with disproportionately larger changes in intra-abdominal fat following endurance exercises of about one hour, thrice weekly (Liao et al, 2002). The addition of aerobic exercises to diet intervention augmented abdominal fat loss, causing a $13 \%$ reduction in visceral adipose tissue, compared with a $7.5 \%$ reduction with diet-alone intervention (Giannopoulou et al, 2005). This is because exercise alters adipose tissue metabolism in individuals, possibly by mobilizing free fatty acids from visceral adipose tissues (Giannopoulou et al, 2005). The increase in body fat noticed in this study during the withdrawal phase, followed the principle of reversibility of effects, because the role of exercises in mobilizing free fatty acids was withdrawn. 
The probable reason for the correlation between some adiposity variables and age of participant and duration of diagnosis could be because aging and long duration of diagnosis is linked with worsening in adiposity variables. Studies mainly carried out in young to middle-aged populations, suggest that $\mathrm{WC}$ is a better anthropometric guide to metabolic risk factor status than BMI, and that WC and not BMI explains obesity-related health risks in this age group (Zhu et al, 2002; Janssen et al, 2004). Wannamethee et al (2005) however claimed that in the elderly, BMI and WC are the best measures of adiposity for predicting the presence of metabolic risk, and suggest that WC can be used as a complementary measurement to identify health risks in normal-weight and overweight elderly persons. In this study, however, it was discovered that during the withdrawal from exercise period, an increase in WC was significantly and directly related to both the age of the participant and the duration of diagnosis of T2D, while an increase in BMI was only directly related to age. We however found that during the same period, PBF, a measure of general adiposity (Wannamethee et al, 2005), was inversely related to both the age of the participant and the duration of diagnosis. This indicates the possibility that $\mathrm{T} 2 \mathrm{D}$ patients actually experience a reduction in their general adiposity as the disorder advances. This may require further confirmation. According to Ramsay et al (2006), simple measures of overweight, such as BMI and WC, are good indicators of the likelihood of morbidity, and prevention of weight gain as age advances is likely to reduce morbidity and disability among older adults.

A larger sample size would probably have provided increased statistical information to reveal stronger correlations, but this was not possible because only individuals who would be able to participate till the end of the $24^{\text {th }}$ week were recruited. A major strength of this study however was that we were able to discover that an increase in adiposity also occurs in patients with T2D, following withdrawal from exercises. Most studies (Klem et al, 1997; Schoeller et al, 1997; Craig, 2007; Jakicic et al, 2008) in this area focussed only on non-diabetic, overweight and obese individuals, with little attention paid to diabetic individuals.

The clinical implication of this study is that T2D patients should be encouraged to participate consistently in therapeutic exercises, considering the fact that a withdrawal leads to a relapse in the hitherto achieved benefits. The possibility of an increase in most of the adiposity variables is higher in older patients and those with a long-standing history of diabetes. Further research should be carried out specifically on how long the effects of exercises are sustained and what factors could lead to withdrawal from exercises.

\section{References}

Craig, J. (2007) How to maintain lost weight. Diabetes Spectrum 20, pp.186-188.

Devlin, M.J., Yanovski, S.Z. and Wilson, G.T. (2000) Obesity: what mental health professionals need to know. American Journal of Psychiatry 157, pp.854-866.

Donaghue, K.C., Fairchild, J.M., Craig, M.E., Chan, A.K., Hing, S., Cutler, L.R., Howard, N.J. and Silink, M. (2003) Do all pre-pubertal years of diabetes duration contribute equally to diabetes complications? Diabetes Care 26, pp.1224-1229.

Fujimoto, W.Y., Jablonski, K.A., Bray, G.A., Kriska, A., BarrettConnor, E., Haffner, S., Hanson, R., Hill, J.O., Hubbard, V., Stamm, E., and Pi-Sunyer, F.X. (2007) Body size and shape changes and the risk of diabetes in the Diabetes Prevention Program. Diabetes 56:1680-1685.

Giannopoulou, I., Ploutz-Snyder, L.L., Carhart, R., Weinstock, R.S., Fernhall, B., Goulopoulou, S. and Kanaley, J.A. (2005) Exercise is required for visceral fat loss in postmenopausal women with type 2 diabetes. The Journal of Clinical Endocrinology and Metabolism 90, pp.1511-1518.

Jakicic, J.M., Marcus, B.H., Lang, W., Janney, C. (2008) Effect of exercise on 24-month weight loss maintenance in overweight women. Archives of Internal Medicine 168, pp.1550-1559.

Janssen, I., Katzmarzyk, P.T. and Ross, R. (2004) Waist circumference and not body mass index explains obesityrelated health risk. American Journal of Clinical Nutrition 79, pp.379-384.

Kengne, A.P., Amoah, A.G.B. and Mbanya, J.C. (2005) Cardiovascular complications of diabetes mellitus in subSaharan Africa. Circulation 112, pp.3592-3601.

King, H. and Herman, W.H. (1998) Global burden of diabetes 1995-2025. Diabetes Care 21, pp.1414-1431.

Klem, M.L., Wing, R.R., McGuire, M.T., Seagle, H.M. and Hill, J.O. (1997) A descriptive study of individuals successful at long-term maintenance of substantial weight loss. American Journal of Clinical Nutrition 66, pp.239-246.

Liao, D., Asberry, P.J., Shofer, J.B., Callahan, H., Matthys, C., Boyko, E.J., Leonetti, D., Kahn, S.E., Austin, M., Newell, L., Schwartz, R.S. and Fujimoto, W.Y. (2002) Improvement of BMI, body composition, and body fat distribution with lifestyle modification in Japanese-Americans with impaired glucose tolerance. Diabetes Care 25, pp.1504-1510.

Martin, M.V., Gomez, G.B., Antoranz, G.M., Fernandez, H.S., Gomez, D.L. and de Oya, O.M. (2001) Validation of the Omron BF 300 monitor for measuring body fat by bioelectric impedance. Aten Primaria 28, pp.174-181. 
Ramsay, S.E., Whincup, P.H., Shaper, A.G. and Wannamethee, S.G. (2006) The relations of body composition and adiposity measures to ill health and physical disability in elderly men. American Journal of Epidemiology 164, pp.459-469.

Scanlon, P.H., Foy, C., Malhotra, R. and Aldington, S.J. (2005) The influence of age, duration of diabetes, cataract and pupil size on image quality in digital photographic retinal screening. Diabetes Care 28, pp.2448-2453.

Schoeller, D.A., Shay, K. and Kushner, R.F. (1997) How much physical activity is needed to minimize weight gain in previously obese women? American Journal of Clinical Nutrition 66, pp.551-556.

Sigal, R.J., Kenny, G.P., Wasserman, D.H. and CastanedaSceppa, C. (2004) Physical activity/exercise and type 2 diabetes. Diabetes Care 27, pp.2518-2539.

Snitker, S., Le, K.Y., Hager, E., Caballero, B. and Black, M.M.
(2007) Association of physical activity and body composition with insulin sensitivity in a community sample of adolescents. Archives of Pediatric and Adolescent Medicine 161, pp.677-683.

Wannamethee, S.G., Shaper, A.G., Morris, R.W. and Whincup, P.H. (2005) Measures of adiposity in the identification of metabolic abnormalities in elderly men. American Journal of Clinical Nutrition 81, pp.1313-1321.

Zhu, S., Wang, Z., Heshka, S., Heo, M., Faith, M.S. and Heymsfield, S.B. (2002) Waist circumference and obesityassociated risk factors among whites in the Third National Health and Nutrition Examination Survey: clinical action thresholds. American Journal of Clinical Nutrition 76, pp.74374. 